\title{
Cosmological Evolution of Supermassive Black Holes: Mass Functions and Spins
}

\author{
Yan-Rong $\mathbf{L i}^{1}$, Jian-Min Wang ${ }^{1}$ and Luis C. $\mathbf{H o}^{2}$ \\ ${ }^{1}$ Key Laboratory for Particle Astrophysics, Institute of High Energy Physics, \\ 19B Yuquan Road, Beijing 100049, China \\ email: liyanrong@mail.ihep.ac.cn, wangjm@mail.ihep.ac.cn \\ ${ }^{2}$ The Observatories of the Carnegie Institution of Washington, \\ 813 Santa Barbara St., Pasadena, CA 91101, USA \\ email: 1ho@obs.carnegiescience.edu
}

\begin{abstract}
We derive the mass function of supermassive black holes (SMBHs) over the redshift range $0<z \lesssim 2$, using the latest deep luminosity and mass functions of field galaxies. Applying this mass function, combined with the bolometric luminosity function of active galactic nuclei (AGNs), into the the continuity equation of SMBH number density, we explicitly obtain the mass-dependent cosmological evolution of the radiative efficiency for accretion. We suggest that the accretion history of SMBHs and their spins evolve in two distinct regimes: an early phase of prolonged accretion, plausibly driven by major mergers, during which the black hole spins up, then switching to a period of random, episodic accretion, governed by minor mergers and internal secular processes, during which the hole spins down. The transition epoch depends on mass, mirroring other evidence for "cosmic downsizing" in the AGN population.
\end{abstract}

Keywords. Black hole physics, galaxy: evolution, quasars: general

\section{Introduction}

It has been realized that SMBHs assemble their mass predominantly through accretion; however, how SMBHs are fueled remains a unsolved issue. The spin of SMBHs traces the angular momentum of the accreted material and therefore is a powerful cosmic probe of SMBH feeding. The link between the radiative efficiency for accretion and black hole spin allows us to analyze the net angular momentum of the accreted gas by quantifying the radiative efficiency. The previous study by Wang et al. (2009) on the cosmological evolution of the radiative efficiency had shown that SMBHs are spinning down with time since $z \approx 2$, strongly implying random accretion onto SMBHs.

Starting from the continuity equation for SMBH mass function, with the help of the duty cycle of SMBHs $\delta\left(t, M_{\bullet}\right)$ and the mean mass accretion rate $\left\langle\dot{M}_{\bullet}\right\rangle=\delta \dot{M}_{\bullet}$, we can obtain the the radiative efficiency from observables by (see Li et al. 2012 for details)

$$
\eta^{-1}\left(z, M_{\bullet}\right)=1+\frac{c^{2}}{\dot{u}\left(z, M_{\bullet}\right)}\left(\frac{\mathrm{d} t}{\mathrm{~d} z}\right)^{-1} \frac{\partial}{\partial z} \int_{M_{\bullet}}^{\infty} N\left(z, M_{\bullet}^{\prime}\right) \mathrm{d} M_{\bullet}^{\prime},
$$

where $\dot{u}\left(z, M_{\bullet}\right)=\bar{\lambda} L_{\mathrm{Edd}} N_{\mathrm{AGN}}, \bar{\lambda}$ is the mean Eddington ratio, $L_{\mathrm{Edd}}$ is the Eddington luminosity, and $N\left(z, M_{\bullet}\right)$ consists of the SMBH mass functions in galaxies $\left(N_{\mathrm{G}}\right)$ and AGNs $\left(N_{\mathrm{AGN}}\right)$. This equation is the generalized $\eta$-equation of Wang et al. (2009).

\section{SMBH mass functions}

We derive the $\mathrm{SMBH}$ mass functions in normal galaxies from the galaxy luminosity function (LF; Cirasuolo et al. 2010) and stellar mass function (SMF; Pérez-González et al. 


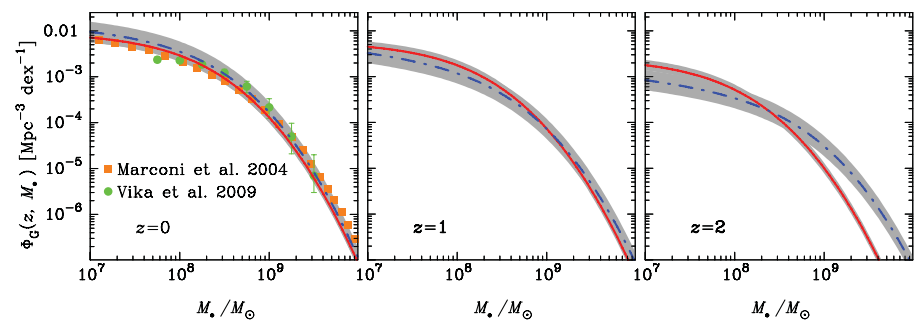

Figure 1. SMBH mass function in normal galaxies at $z=0,1$, and 2 , derived from the galaxy LF (red solid lines) and the galaxy SMF (blue dot-dashed lines).

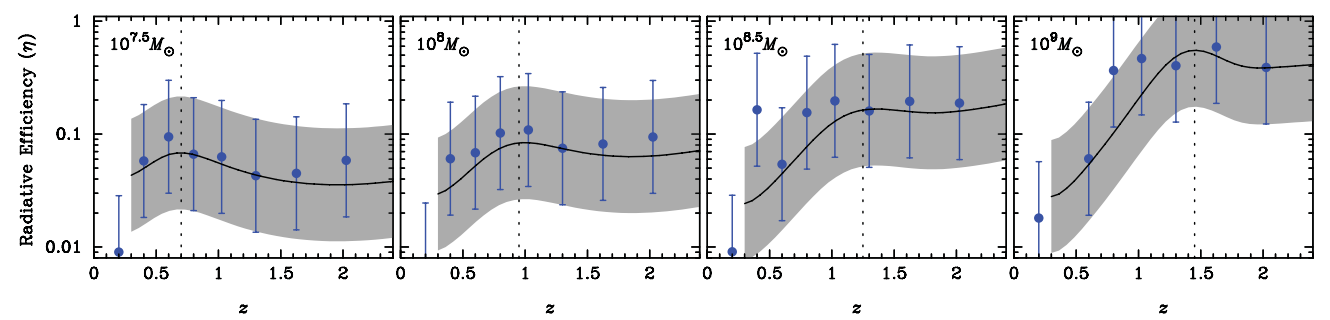

Figure 2. Radiative efficiency evolution for different black hole mass. Solid lines and data points are the efficiency using the SMBH mass function derived from the galaxy LF and SMF, respectively.

2008), respectively. We use three ingredients: (1) the bulge-to-total luminosity ratio; (2) a relation between $\mathrm{SMBH}$ and spheroid mass; and, for the method involving the galaxy LF, (3) a prescription to describe the passive evolution of the spheroid luminosity. Fig. 1 shows the obtained SMBH mass functions at $0<z \lesssim 2$. A good agreement between the two methods can be found ( $\mathrm{Li}$ et al. 2011).

The SMBH mass function of AGNs is calculated by combining the observed Eddington ratio distribution and the AGN bolometric LF of Hopkins et al. (2007).

\section{Evolution of the radiative efficiency and SMBH spins}

The radiative efficiency evolution for different black hole masses is plotted in Fig. 2. Assuming that the radiative efficiency provides an effective indirect measure of the black hole spin, we propose that: (1) The accretion history of SMBHs and their spin evolution can be characterized by two regimes: an initial phase of mass accumulation from prolonged accretion that spins up the hole, followed by a period of random, episodic accretion that spins down the hole toward lower redshifts (Wang et al. 2009). (2) The evolution of the spin, like the global pattern of AGN activity, exhibits "cosmic downsizing". Highmass holes gain their masses earlier, reach the peak of their AGN activity earlier, and begin to spin down earlier. Random accretion dominates their evolution below $z \approx 2$, whereas lower mass holes transition to this phase later, at $z \approx 1$ (Li et al. 2012).

\section{References}

Cirasuolo, M., et al. 2010, MNRAS, 401, 1166

Hopkins, P. F., Richards, G. T., \& Hernquist, L. 2007, ApJ, 654, 731

Li, Y.-R., Ho, L. C., \& Wang, J.-M. 2011, ApJ, 742, 33

Li, Y.-R., Wang, J.-M., \& Ho, L. C. 2012, ApJ, 749, 187

Pérez-González, P. G., et al. 2008, ApJ, 675, 234

Wang, J.-M., et al. 2009, ApJ, 697, L141 